\title{
The Tanh Methods for the Hirota Equations
}

\author{
Kamal Raslan \\ Department of Mathematics \\ Science \\ Al-Azhar University, Egypt
}

\begin{abstract}
In this paper we applied the tanh method for analytic study of the nonlinear equations of partial differential equations(PDEs).The proposed method gives more general exact traveling wave solutions without much extra effort. Three applications from literature of nonlinear equation of PDEs were solved by the method. The calculations demonstrate the effectiveness and convenience of the method for nonlinear sub system of PDEs.
\end{abstract}

\section{Keywords}

Tanh method, Hirota equations, exact solutions.

\section{INTRODUCTION}

The nonlinear partial differential equations (NPDEs) are widely used to describe many important phenomena and dynamic processes in physics, chemistry, biology, fluid dynamics, plasma, optical fibers and other areas of engineering. Many efforts have been made to study NPDEs. One of the most exciting advances of nonlinear science and theoretical physics has been a development of methods that look for exact solutions for nonlinear evolution equations. The availability of symbolic computations such as Mathematica program has popularized direct seeking for exact solutions of nonlinear equations. Therefore, exact solution methods of nonlinear evolution equations have become more and more important resulting in methods like the tanh method [1-3], extended tanh function method [4], the modified extended tanh function method [5], the generalized hyperbolic function [6].Most of exact solutions have been obtained by these methods, including the solitary wave solutions, shock wave solutions, periodic wave solutions, and the like.

In this paper, we propose tanh-coth and tan-cot, methods to obtain an exact single-soliton and travelling wave solutions of the Hirota equation with a source. In order to illustrate the effectiveness and convenience of the method, we consider the Hirota equation in the form $[12,13]$, The standard tanh method and the proposed modifications all depend on the balance method, where the linear terms of highest order are balanced with the highest order nonlinear terms of the reduced equation. In this paper, we use the tanh method to find the exact solutions of the following nonlinear PDEs: the nonlinear equation of the Hirota equations. This will be useful in numerical studies.

\section{OUTLINE OF THE TANH \& TAN METHODS}

The tanh method will be introduced as presented by Malfliet [8] and by Wazwaz [9-11]. The tanh method is based on a priori assumption that the traveling wave solutions can be expressed in terms of the tanh function to solve the coupled $\mathrm{KdV}$ equations. The tanh method is developed by Malfliet [8]. The method is applied to find out an exact solution of a nonlinear ordinary differential equation. Consider the following PDE.

Consider the nonlinear partial differential equation in the form

$F\left(u, u_{t}, u_{x}, u_{x x}, u_{x x x}, \ldots\right)=0$

\author{
Zain F. Abu Shaeer
}

Where $u(x, t)$ is the solution of nonlinear partial differential equation Eq. (1). We use the transformations, $=\kappa(x-\lambda t)$, to transform $u(x, t)$ to $U(\xi)$ give :

$$
\frac{\partial}{\partial \mathrm{t}}=-\kappa \lambda \frac{d}{d \xi}, \frac{\partial}{\partial \mathrm{x}}=\kappa \frac{d}{d \xi}, \frac{\partial^{2}}{\partial \mathrm{x}^{2}}=\kappa^{2} \frac{d^{2}}{d \xi^{2}}, \frac{\partial^{3}}{\partial \mathrm{x}^{3}}=\kappa^{3} \frac{d^{3}}{d \xi^{3}} \text {, and }
$$

so on,then Eq.(1) becomes an ordinary differential equation

$$
N\left(U, \kappa \lambda U^{\prime}, \kappa U^{\prime}, \kappa^{2} U^{\prime \prime}, \kappa^{3} U^{\prime \prime \prime}, \ldots\right)=0,
$$

With $\mathrm{N}$ being another polynomial form of its argument, which will be called the reduced ordinary differential equation of Eq. (2). Integrating Eq.(2) as long as all terms contain derivatives, the integration constants are considered to be zeros in view of the localized solutions. However, the nonzero constants can be used and handled as well [11]. Now finding the traveling wave solutions to Eq. (1) is equivalent to obtaining the solution to to the reduced ordinary differential equation (2). For the tanh method, we introduce the new independent variable [13]

$$
\mathrm{Y}(\mathrm{x}, \mathrm{t})=\tanh (\xi) \text {, or } \mathrm{Y}(\mathrm{x}, \mathrm{t})=\operatorname{coth}(\xi),
$$

that leads to a change in the derivatives:

$$
\begin{aligned}
& \frac{\mathrm{d}}{\mathrm{d} \xi}=\left(1-Y^{2}\right) \frac{\mathrm{d}}{\mathrm{d} Y} \\
& \frac{d^{2}}{d \xi^{2}}=\left(1-Y^{2}\right)\left[-2 Y \frac{d}{d Y}+\left(1-Y^{2}\right) \frac{d^{2}}{d Y^{2}}\right] \\
& \frac{d^{3}}{d \xi^{3}}=\left(1-Y^{2}\right)\left[2\left(3 Y^{2}-1\right) \frac{d}{d Y}-6 Y\left(1-Y^{2}\right) \frac{d^{2}}{d Y^{2}}+\right. \\
& \left.\left(1-Y^{2}\right)^{2} \frac{d^{3}}{d Y^{3}}\right]
\end{aligned}
$$

Where the other derivatives can be derived in a similar way. We use new independent variables

$$
Y(x, t)=\tan (\xi) \text {, or } Y(x, t)=\cot (\xi),
$$

that leads to the change of derivatives

$$
\begin{aligned}
& \frac{d}{d \xi}=\left(1+Y^{2}\right) \frac{d}{d Y} \\
& \frac{d^{2}}{d \xi^{2}}=\left(1+Y^{2}\right)\left[-2 Y \frac{d}{d Y}+\left(1+Y^{2}\right) \frac{d^{2}}{d Y^{2}}\right] \\
& \frac{d^{3}}{d \xi^{3}}=\left(1+Y^{2}\right)\left[2\left(3 Y^{2}-1\right) \frac{d}{d Y}-6 Y\left(1+Y^{2}\right) \frac{d^{2}}{d Y^{2}}+\right. \\
& \left.\left(1+Y^{2}\right)^{2} \frac{d^{3}}{d Y^{3}}\right]
\end{aligned}
$$

The next crucial step is that the solution we are looking for is expressed in the form

$$
u(x, t)=U(\xi)=\sum_{i=1}^{m} a_{i} Y^{i}=a_{0}+\cdots+a_{m} Y^{m},
$$

where the parameter $m$ can be found by balancing the highestorder linear term with the

nonlinear terms in Eq. (2), and $\mathrm{k}, \lambda, a_{0}, a_{1}, \ldots \ldots, a_{m}$ are to be determined. Substituting (5) into (2) will yield a set of algebraic equations $k, \lambda, a_{0}, a_{1}, \ldots \ldots, a_{m}$ because all coefficients of $Y^{i}$ have to vanish. From these relations, $\mathrm{k}, \lambda, a_{0}, a_{1}, \ldots ., a_{m}$ can be obtained. Having determined these parameters, knowing that $m$ is a positive integer in most cases, and using (5) we obtain an analytic solution $u(x, t)$ in 
a closed form [11].The tanh method seems to be powerful tool in dealing with coupled nonlinear physical models. For a coupled system of nonlinear differential equations with two unknowns:

$$
\begin{aligned}
& F_{1}\left(u, v, u_{t}, v_{t}, u_{x}, v_{x}, u_{x x}, v_{x x}, \ldots\right)=0 \\
& F_{2}\left(u, v, u_{t}, v_{t}, u_{x}, v_{x}, u_{x x}, v_{x x}, \ldots\right)=0
\end{aligned}
$$

As for the traveling wave solutions to (6) concerned, we have to solve its corresponding reduced ordinary differential equations

$N_{1}\left(u, v, u^{\prime}, v^{\prime}, u^{\prime \prime}, v^{\prime \prime}, \ldots\right)=0$

$N_{2}\left(u, v, u^{\prime}, v^{\prime}, u^{\prime \prime}, v^{\prime \prime}, \ldots\right)=0$,

In most cases, the exact solvability of (7) depends on a delicate explicit assumption between the two unknowns or their derivatives, for more details see [12].

\section{NUMERICAL EXAMPLES}

The tanh method is generalized on three examples including Hirota equations.

\section{Example 1.}

Let us consider the Hirota equation(1) in the form [12]

$$
i u_{t}+u_{x x}+2|u|^{2} u+i \alpha u_{x x x}+6 i \alpha|u|^{2} u_{x} \text {, }
$$

Which is the standard Schrödinger equation in the case when $\alpha=0$, is a famous mathematical and physical equation (Example(3)). Where the cubic term in Eq. (10) describes the nonlinear-self interaction in the high frequency subsystem, such a term corresponds to a self-focusing effect in plasma physics. The coefficient $\alpha$ is a real constant that can be a positive or negative number.

$u(x, t)=u_{1}(x, t)+i u_{2}(x, t)$,

Using the traveling wave transformations.

$$
\begin{aligned}
& u_{1}(x, t)=U(\xi)=\sum_{i=1}^{m} a_{i} Y^{i}, \\
& u_{2}(x, t)=V(\xi)=\sum_{i=1}^{m} b_{i} Y^{i}, \xi=\kappa(x-\lambda t),
\end{aligned}
$$

The nonlinear system of partial differential equations (10) is carried to a system of ordinary differential equations.

$$
\begin{aligned}
& \lambda \kappa \frac{d V}{d \xi}+2\left(V^{2}+U^{2}\right) U+\kappa^{2} \frac{d^{2} U}{d \xi^{2}}-\alpha \kappa^{3} \frac{d^{3} V}{d \xi^{3}} \\
& -6 \alpha \kappa\left(V^{2}+U^{2}\right) \frac{d V}{d \xi}=0, \\
& \lambda \kappa \frac{d U}{d \xi}-2\left(V^{2}+U^{2}\right) V-\kappa^{2} \frac{d^{2} V}{d \xi^{2}}-\alpha \kappa^{3} \frac{d^{3} U}{d \xi^{3}}-6 \alpha \kappa\left(V^{2}+\right. \\
& \left.U^{2}\right) \frac{d U}{d \xi}=0,
\end{aligned}
$$

We postulate the following tanh series in Eq. (11), Eq. (3) and the transformation given in (4), the equation in (13) reduces to

$$
\begin{aligned}
& \lambda \kappa\left(1-Y^{2}\right) \frac{d V}{d Y}+2\left(V^{2}+U^{2}\right) U+\kappa^{2}\left(1-Y^{2}\right)\left[-2 Y \frac{d U}{d Y}+\right. \\
& \left.\left(1-Y^{2}\right) \frac{d^{2} U}{d Y^{2}}\right]-\alpha \kappa^{3}\left(1-Y^{2}\right)\left[2\left(3 Y^{2}-1\right) \frac{d V}{d Y}-\right. \\
& \left.6 Y\left(1-Y^{2}\right) \frac{d^{2} V}{d Y^{2}}+\left(1-Y^{2}\right)^{2} \frac{d^{3} V}{d Y^{3}}\right]-6 \alpha \kappa\left(V^{2}+U^{2}\right)(1- \\
& \left.Y^{2}\right) \frac{d V}{d Y}=0, \\
& -\lambda \kappa\left(1-Y^{2}\right) \frac{d U}{d Y}+2\left(V^{2}+U^{2}\right) V+\kappa^{2}\left(1-Y^{2}\right)\left[-2 Y \frac{d V}{d Y}+\right. \\
& \left.\left(1-Y^{2}\right) \frac{d^{2} V}{d Y^{2}}\right]+\alpha \kappa^{3}\left(1-Y^{2}\right)\left[2\left(3 Y^{2}-1\right) \frac{d U}{d Y}-\right. \\
& \left.6 Y\left(1-Y^{2}\right) \frac{d^{2} U}{d Y^{2}}+\left(1-Y^{2}\right)^{2} \frac{d^{3} U}{d Y^{3}}\right]+6 \alpha \kappa\left(V^{2}+U^{2}\right)(1- \\
& \left.Y^{2}\right) \frac{d U}{d Y}=0,
\end{aligned}
$$

Now, to determine the parameters $m$ and $n$, we balance the linear term of highest-order with the highest order nonlinear terms. So, in Eq. (14) we balance $V^{\prime \prime \prime}$ with $U^{2} V^{\prime}$, to obtain $2 m=2$, then $m=1$.while in Eq. (15) we balance $U^{\prime \prime \prime}$ with $V^{2} U^{\prime}$, to obtain

$2 n=2$, then $n=1$.The tanh method admits the use of the finite expansion for both

$$
\begin{array}{ll}
u_{1}(x, t)=U(Y)=a_{0}+a_{1} Y, & a_{1} \neq 0, \\
u_{2}(x, t)=V(Y)=b_{0}+b_{1} Y, & b_{1} \neq 0,
\end{array}
$$

Substituting $U, U^{\prime}, U^{\prime \prime}, U^{\prime \prime \prime}$ and $V, V^{\prime}, V^{\prime \prime}, V^{\prime \prime \prime}$ from Eq. (16) and Eq. (17) respectively into Eqs.(14-15), then equating the coefficient of $Y^{i}, i=0,1,2,3,4$ leads to the following nonlinear system of algebraic equations.

$Y^{0}$ Coeff

$2 a_{0}^{3}+2 a_{0} b_{0}^{2}-6 a_{0}^{2} b_{0} \alpha \kappa-6 b_{0}^{2} b_{1} \alpha \kappa+2 b_{1} \alpha \kappa^{3}+b_{1} \kappa \lambda$ $=0$

$Y^{1}$ Coeff.

$a_{0}^{2} a_{1}+a_{1} b_{0}^{2}+2 a_{0} b_{0} b_{1}-6 a_{0} a_{1} b_{1} \alpha \kappa-6 b_{0} b_{1}^{2} \alpha \kappa$ $-a_{1} \kappa^{2}=0$

$Y^{2}$ Coeff.

$6 a_{0} a_{1}^{2}+4 a_{1} b_{0} b_{1}+2 a_{0} b_{1}^{2}+6 a_{0}^{2} b_{1} \alpha \kappa-6 a_{1}^{2} b_{1} \alpha \kappa+$ $6 b_{0}^{2} b_{1} \alpha \kappa-6 b_{1}^{3} \alpha \kappa-8 b_{1} \alpha \kappa^{3}-b_{1} \kappa \lambda=0$

$Y^{3}$ Coeff.

$a_{1}^{3}+a_{1} b_{1}^{2}+6 a_{0} a_{1} b_{1} \alpha \kappa+6 b_{0} b_{1}^{2} \alpha \kappa+a_{1} \kappa^{2}=0$

$Y^{4}$ Coeff. $a_{1}^{2}+b_{1}^{2}+\kappa^{2}=0$,

and

$Y^{0}$ Coeff.

$2 a_{0}^{2} b_{0}+2 b_{0}^{3}+6 a_{0}^{2} a_{1} \alpha \kappa+6 a_{1} b_{0}^{2} \alpha \kappa-2 a_{1} \alpha \kappa^{3}-a_{1} \kappa \lambda$ $=0$,

$Y^{1}$ Coeff.

$2 a_{0} a_{1} b_{0}+a_{0}^{2} b_{1}+3 b_{0}^{2} b_{1}+6 a_{0} a_{1}^{2} \alpha \kappa+6 a_{1} b_{0} b_{1} \alpha \kappa$ $-b_{1} \kappa^{2}=0$,

$Y^{2}$ Coeff.

$2 a_{1}^{2} b_{0}+4 a_{0} a_{1} b_{1}+6 b_{0} b_{1}^{2}-6 a_{0}^{2} a_{1} \alpha \kappa+6 a_{1}^{3} \alpha \kappa-$ $6 a_{1} b_{0}^{2} \alpha \kappa+6 a_{1} b_{1}^{2} \alpha \kappa+8 a_{1} \alpha \kappa^{3}+a_{1} \kappa \lambda=0$,

$Y^{3}$ Coeff.

$b_{1}^{3}+a_{1}^{2} b_{1}-6 a_{0} a_{1}^{2} \alpha \kappa-6 b_{0} a_{1} b_{1} \alpha \kappa+b_{1} \kappa^{2}=0$,

$Y^{4}$ Coeff. $-b_{1}^{2}-a_{1}^{2}-\kappa^{2}=0$,

Solving the nonlinear systems of equations (18) with help of Mathematica we can get:

Case(1)

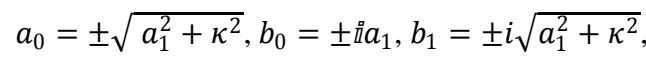

and $\lambda=2\left(2 \alpha \kappa^{2} \pm \stackrel{\pi}{\Pi} \kappa\right)$,

The kink solitons solutions (1) take the forms,

$u(x, t)=(1 \pm \tanh (k(x-\lambda t)))\left(\sqrt{a_{1}^{2}+\kappa^{2}} \pm a_{1}\right)$, and

$u(x, t)=(1 \pm \operatorname{coth}(k(x-\lambda t)))\left(\sqrt{a_{1}^{2}+\kappa^{2}} \pm a_{1}\right)$, 


\section{Case(2)}

$\lambda=2\left(2 \alpha \kappa^{2} \pm \stackrel{a}{\amalg} \kappa\right), a_{0}= \pm \kappa, a_{1}=0, b_{0}=0$,

and $b_{1}= \pm i \kappa$;

The kink solitons solutions(2) take the forms,

$$
\begin{aligned}
& u(x, t)= \pm \kappa(1+\tanh (k(x-\lambda t))), \text { and } \\
& u(x, t)= \pm \kappa(1+\operatorname{coth}(k(x-\lambda t))),
\end{aligned}
$$

The solitary wave and behavior of the solutions $u_{1}(x, t)$, $u_{2}(x, t)$ and $u(x, t)$ are shown in Figure (1) for some fixed values of the parameters, $\left(a_{1}=2, \alpha=1, \kappa=0.5\right)$
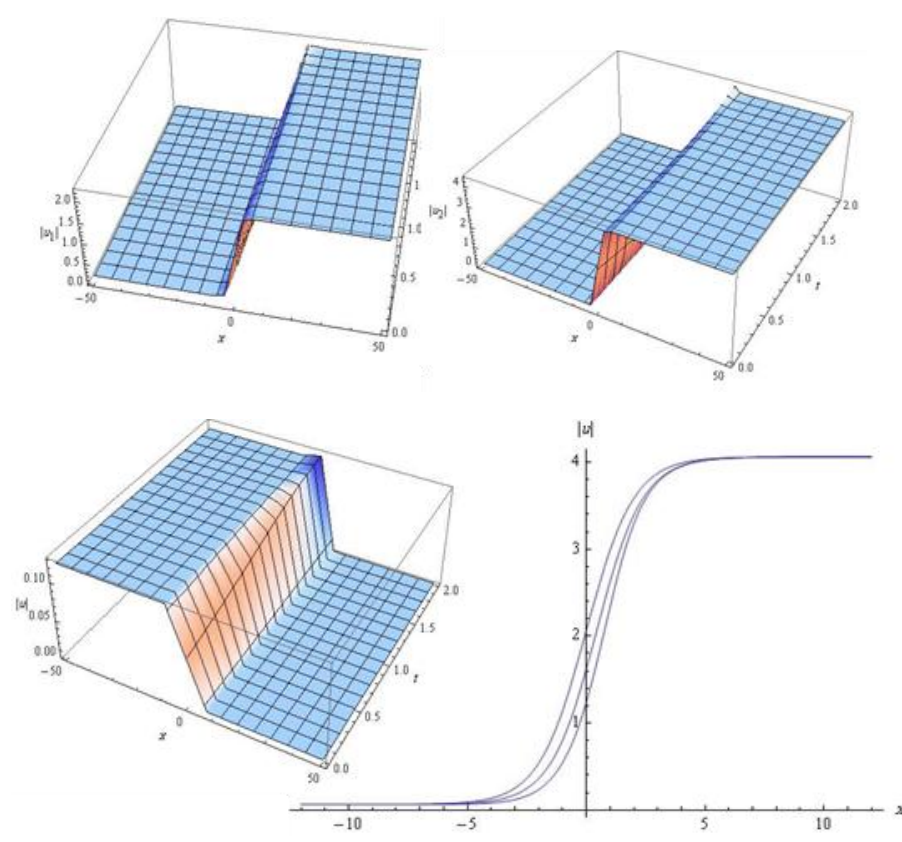

Fig 1: the component $\left|u_{1}\right|$, the component $\left|u_{2}\right|$, and $|u|$ of the example(1)

\section{Example 2}

The Hirota Equation(2) [12]

$$
q_{t}+3 \alpha|q|^{2} q_{x}+\gamma q_{x x x}=0
$$

Where $q$ is a complex valued function of the spatial coordinate $x$ and the time $t, \alpha$ and $\gamma$ are positive real constants. This equation is an integrable equation which has a number of physical applications, such as the propagation of optical pluses in nematic liquid crystal waveguides. The Hirota equation is closely related to both the nonlinear Schrodinger (NLS) and modified Korteweg-de Vries (mKdV) equations,as it is complex generalization of the $\mathrm{mKdV}$ equation and it is a part of the NLS hierarchy of the integrable equation. Also, its soliton solution has a very similar form to the NLS soliton.The Hirota equation (22) has a two-parameter soliton family, with amplitude and velocity

To avoid complex computation we assume

$q(x, t)=u(x, t)+i v(x, t), \quad i^{2}=-1$,

where $u(x, t), v(x, t)$ are real functions. This will reduce Hirota equation to the coupled system

$$
\begin{gathered}
-\lambda \frac{d U}{d \xi}+3 \alpha\left(V^{2}+U^{2}\right) \frac{d U}{d \xi}+\gamma \kappa^{2} \frac{d^{3} U}{d \xi^{3}}=0, \\
-\lambda \frac{d V}{d \xi}+3 \alpha\left(V^{2}+U^{2}\right) \frac{d V}{d \xi}+\gamma \kappa^{2} \frac{d^{3} V}{d \xi^{3}}=0,
\end{gathered}
$$

We postulate the following tanh series in Eq. (4), Eq. (23) and the transformation, the equation (24) reduces to

$-\lambda\left(1-Y^{2}\right) \frac{d U}{d Y}+3 \alpha\left(V^{2}+U^{2}\right)\left(1-Y^{2}\right) \frac{d U}{d Y}+\gamma \kappa^{2}(1-$
$\left.Y^{2}\right)\left[2\left(3 Y^{2}-1\right) \frac{d U}{d Y}-6 Y\left(1-Y^{2}\right) \frac{d^{2} U}{d Y^{2}}+\left(1-Y^{2}\right)^{2} \frac{d^{3} U}{d Y^{3}}\right]=$

0 ,

$-\lambda\left(1-Y^{2}\right) \frac{d V}{d Y}+3 \alpha\left(V^{2}+U^{2}\right)\left(1-Y^{2}\right) \frac{d V}{d Y}+\gamma \kappa^{2}(1-$

$\left.Y^{2}\right)\left[2\left(3 Y^{2}-1\right) \frac{d V}{d Y}-6 Y\left(1-Y^{2}\right) \frac{d^{2} V}{d Y^{2}}+\left(1-Y^{2}\right)^{2} \frac{d^{3} V}{d Y^{3}}\right]=$

0 ,

Now, determine the parameters, we find $m=n=1$. The tanh method admits the use of the finite expansion for both

$$
\begin{array}{ll}
u(x, t)=U(Y)=a_{0}+a_{1} Y, & a_{1} \neq 0, \\
v(x, t)=V(Y)=b_{0}+b_{1} Y, & b_{1} \neq 0,
\end{array}
$$

Substituting $U, U^{\prime}, U^{\prime \prime}, U^{\prime \prime \prime}$ and,$V^{\prime \prime}$ from Eq. (27) and Eq. (28) respectively into Eq. $(25,26)$, then equating the coefficient of $Y^{i}, i=0,1,2,3,4$ leads to the following nonlinear system of algebraic equations

$Y^{0}$ Coeff. $\quad 3 a_{0}^{2} \alpha+3 b_{0}^{2} \alpha-2 \gamma \kappa^{2}-\lambda=0$

$Y^{1}$ Coeff. $\quad a_{0} a_{1}+b_{0} b_{1}=0$

$Y^{2}$ Coeff. $-3 a_{0}^{2} \alpha+3 a_{1}^{2} \alpha-3 b_{0}^{2} \alpha+3 b_{1}^{2} \alpha+8 \gamma \kappa^{2}+\lambda=0$

$Y^{3}$ Coeff. $-a_{0} a_{1}-b_{0} b_{1}=0$

$Y^{4}$ Coeff. $-a_{1}^{2} \alpha-b_{1}^{2} \alpha-2 \gamma \kappa^{2}=0$,

Solving these systems, We find the kink solitons solutions take the forms.

Case (1)

$\lambda=-2 \gamma \kappa^{2}, \varpi_{1}=\left( \pm \frac{\sqrt{a_{1}^{2} \alpha+2 \gamma \kappa^{2}}}{\sqrt{\alpha}}\right), \quad b_{1}=i \varpi_{1}, b_{0}=0$,

and $\boldsymbol{a}_{\mathbf{0}}=\mathbf{0}$,

$q=\left(a_{1}-\varpi_{1}\right) \tanh (\kappa(x-\lambda t))$, or

$q=\left(a_{1}-\varpi_{1}\right) \operatorname{coth}(\kappa(x-\lambda t))$,

Case(2)

$\lambda=3 \mathbf{b}_{0}^{2} \alpha-2 \gamma \boldsymbol{\kappa}^{2}, \quad a_{1}= \pm \frac{\sharp \sqrt{2} \sqrt{\gamma} \kappa}{\sqrt{\alpha}}, \quad a_{0}=0$, and $b_{1}=\mathbf{0}$,

$q=i b_{0} \pm \frac{I \sqrt{2} \sqrt{\gamma} \kappa}{\sqrt{\alpha}} \tanh (\kappa(x-\lambda t))$, or

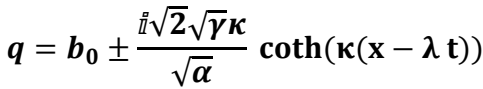

Case(3)

$\lambda=3 a_{0}^{2} \alpha-2 \gamma \kappa^{2}, \quad b_{1}= \pm \frac{i \sqrt{2} \sqrt{\gamma} \kappa}{\sqrt{\alpha}}, b_{0}=0$, and $a_{1}=0$

$q=a_{0} \pm \frac{\tilde{\pi}^{2} \sqrt{2 \gamma}}{\sqrt{\alpha}} \kappa \tanh (\kappa(x-\lambda t))$, or $q=a_{0} \pm$ $\frac{\ddot{\Delta}^{2} \sqrt{2 \gamma}}{\sqrt{\alpha}} \kappa \operatorname{coth}(\kappa(x-\lambda \mathrm{t}))$

Case(4)

$$
\begin{aligned}
& \lambda=-2\left(1+3 a_{0}^{2}\right) \gamma \kappa^{2}, \quad \varpi_{2}=\sqrt{1+\frac{2 \gamma \kappa^{2}}{\alpha}}, \\
& b_{0}= \pm i a_{0} \varpi_{2}, b_{1}=1, \text { and } a_{1}= \pm i \varpi_{2} \\
& q=\left( \pm \varpi_{2}\right)\left(i \tanh (\kappa(x-\lambda t))-a_{0}\right)+ \\
& \left(a_{0}+i \tanh (\kappa(x-\lambda t))\right),
\end{aligned}
$$




\section{In terms tan method}

Similar as the tanh method we can obtain the tan method through the equation (6)

$\operatorname{Case}(\mathbf{1}) \lambda=2\left(\gamma \kappa^{2}-\frac{3 a_{0}^{2} \gamma \kappa^{2}}{b_{1}^{2}}\right), \varpi_{3}= \pm \sqrt{\frac{\left(b_{1}^{2} \alpha+2 \gamma \kappa^{2}\right)}{\alpha}}$,

$a_{1}=i \varpi_{3}, \quad b_{0}=\frac{i a_{0}}{b_{1}} \varpi_{3}$,

$q=a_{0}-\varpi_{3}\left(\frac{a_{0}}{b_{1}}+i \tan (\kappa(x-\lambda \mathrm{t}))\right)+\mathrm{i} b_{1} \tan (\kappa(x-$

$\lambda \mathrm{t})), \quad q=a_{0}-\varpi_{3}\left(\frac{a_{0}}{b_{1}}+i \cot (\kappa(x-\lambda \mathrm{t}))\right)+$

i $b_{1} \cot (\kappa(x-\lambda t))$

\section{Case(2)}

$\lambda=2 \gamma \boldsymbol{\kappa}^{2}, \quad \boldsymbol{a}_{1}=\boldsymbol{i} \varpi_{3}, \boldsymbol{b}_{0}=\mathbf{0}$, and $\boldsymbol{a}_{\mathbf{0}}=\mathbf{0}$,

$q=\boldsymbol{i}\left(b_{1}-\varpi_{3}\right) \tan (\kappa(x-\lambda t)), \quad$ or

$q=i\left(b_{1}-\varpi_{3}\right) \cot (\kappa(x-\lambda t))$,

Case(3)

$\lambda=3 b_{0}^{2} \alpha+2 \gamma \boldsymbol{\kappa}^{2}, \quad a_{1}= \pm \frac{i \sqrt{2} \sqrt{\gamma} \boldsymbol{1}}{\sqrt{\alpha}}, \quad b_{1}=0, \quad$ and

$a_{0}=0$,

$q=i b_{0} \pm \frac{\sharp \pi \sqrt{2} \sqrt{\gamma} \kappa}{\sqrt{\alpha}} \tan (\kappa(x-\lambda t))$, or

$q=i b_{0} \pm \frac{i \sqrt{2} \sqrt{\gamma} \kappa}{\sqrt{\alpha}} \cot (\kappa(x-\lambda t))$,

The solitary wave and behavior of the solutions $u(x, t)$ and $v(x, t)$ are shown in Figure(2) for some fixed values of the parameters, $(\lambda=0.5, \kappa=0.05)$
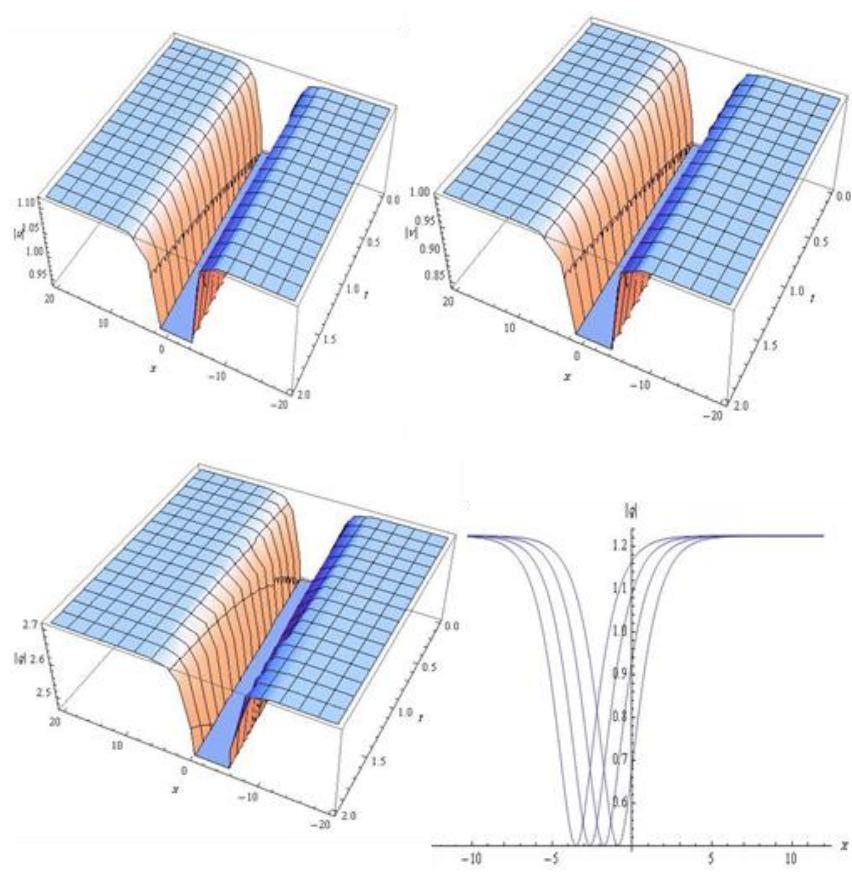

Fig 2: The component $|u|$, the component $|v|$, and $|q|$ of the example(2) in terms tanh method

\section{Example 3.}

The Hirota Equation(3). One of the most important model equations in nonlinear science is the nonlinear Schrodinger (NLS) equation,[14] $i \eta_{t}+\eta_{x x}+\eta|\eta|^{2}=0$,

Physically, the NLS equation describes the modulation of weakly-nonlinear wave trains. In deep water. Benjamin and Feir [Benjamin and Feir(1967)] showed that an uniform wave train is unstable to long wave perturbations. Peregrine [Peregrine(1985)] and Yuen and Lake [Yuen and Lake(1982)] present a historical overview of fluid mechanics applications of the NLS equation and its physical origins. In the optical context, the NLS equation was derived by Hasegawa and Tappert [Hasegawa and Tappert(1973)]. It also describes the evolution of the slowly varying envelope of an optical pulse. Derived asymptotically from Maxwell's equations, it assumes slow variation in the carrier frequency and the Kerr dependence. The NLS equation is central to understanding soliton propagation in optical fibres, which is of critical importance to the field of fibre-based telecommunications Wabnitz,Kodama and Aceves].Motivated by these physical applications,

the evolution of a NLS soliton has been studied extensively in both the physical and mathematical communities.

$$
\eta(x, t)=\varphi(x, t)+i \psi(x, t),
$$

where $\varphi(x, t), \psi(x, t)$ are real functions. This will reduce Hirota equation to the coupled system

$$
\begin{aligned}
& -\lambda \kappa \frac{d \phi}{d \xi}+\left(\Psi^{2}+\phi^{2}\right) \phi+\kappa^{2} \frac{d^{2} \phi}{d \xi^{2}}=0, \\
& -\lambda \kappa \frac{d \Psi}{d \xi}+\left(\Psi^{2}+\phi^{2}\right) \Psi+\kappa^{2} \frac{d^{2} \Psi}{d \xi^{2}}=0,
\end{aligned}
$$

We postulate the following tanh series in Eq. (33), Eq. (3) and the transformation given in (4), the equation (34) reduces to

$$
\begin{aligned}
& -\lambda\left(1-Y^{2}\right) \frac{d \phi}{d Y}+ \\
& \left(\Psi^{2}+\phi^{2}\right)\left(1-Y^{2}\right) \frac{d \phi}{d Y}+\gamma \kappa^{2}\left(1-Y^{2}\right)\left[2\left(3 Y^{2}-1\right) \frac{d \phi}{d Y}-\right. \\
& \left.\quad 6 Y\left(1-Y^{2}\right) \frac{d^{2} \phi}{d Y^{2}}\right]=0, \\
& -\lambda\left(1-Y^{2}\right) \frac{d \Psi}{d Y}+\left(\Psi^{2}+\phi^{2}\right)\left(1-Y^{2}\right) \frac{d \Psi}{d Y}+\kappa^{2}(1- \\
& \left.Y^{2}\right)\left[2\left(3 Y^{2}-1\right) \frac{d \Psi}{d Y}-6 Y\left(1-Y^{2}\right) \frac{d^{2} \Psi}{d Y^{2}}\right]=0,
\end{aligned}
$$

Now, to determine the parameters $m$ and $n$, we balance the linear term of highest-order with the highest order nonlinear terms. then $m=n=1$.The tanh method admits the use of the finite expansion for both

$$
\begin{array}{ll}
\varphi(x, t)=\Phi(Y)=a_{0}+a_{1} Y, \quad a_{1} \neq 0, \\
\psi(x, t)=\Psi(Y)=b_{0}+b_{1} Y, & b_{1} \neq 0,
\end{array}
$$

Substituting from Eq. (37) and Eq. (38) respectively into Eq.(35-36), then equating the coefficient of $Y^{i}, i=0,1,2,3$ leads to the following nonlinear system of algebraic equations

$$
\begin{array}{ll}
Y^{0} \text { Coeff. } & a_{0}^{3}+a_{0} b_{0}^{2}+b_{1} \kappa \lambda=0, \\
Y^{1} \text { Coeff. } & 3 a_{0}^{2} a_{1}+a_{1} b_{0}^{2}+2 a_{0} b_{0} b_{1}-2 a_{1} \kappa^{2}=0, \\
Y^{2} \text { Coeff. } & 6 a_{0} a_{1}^{2}+4 a_{1} b_{0} b_{1}+2 a_{0} b_{1}^{2}-2 b_{1} \kappa \lambda=0, \\
Y^{3} \text { Coeff. } & a_{1}^{2}+b_{1}^{2}+2 \kappa^{2}=0, \\
& \\
Y^{0} \text { Coeff. } & a_{0}^{2} b_{0}+b_{0}^{3}-a_{1} \kappa \lambda=0, \\
Y^{1} \text { Coeff. } & 2 a_{0} a_{1} b_{0}+a_{0}^{2} b_{1}+3 b_{0}^{2} b_{1}-2 b_{1} \kappa^{2}=0,
\end{array}
$$$$
\text { and }
$$ 


$$
\begin{aligned}
& Y^{2} \text { Coeff. } \quad 2 b_{0} a_{1}^{2}+4 a_{0} a_{1} b_{1}+6 b_{0} b_{1}^{2}+2 a_{1} \kappa \lambda=0, \\
& Y^{3} \text { Coeff. } \quad a_{1}^{2}+b_{1}^{2}+2 \kappa^{2}=0,
\end{aligned}
$$

Solving these systems, we find the solitons solutions take the forms

$$
\begin{aligned}
& \text { Case (1) } \\
& \lambda= \pm 2 i \kappa, \quad a_{1}=\left( \pm \frac{\sqrt{2 a_{0}^{2}+\lambda^{2}}}{\sqrt{2}}\right), b_{1}= \pm i a_{0}, \text { and } b_{0}= \pm i a_{1}, \\
& \eta=a_{0}(1 \pm i \tanh (\kappa(\mathrm{x} \pm 2 i \kappa t))) \pm \sqrt{\frac{2 a_{0}^{2}+\lambda^{2}}{2}}(\tanh (\mathrm{x} \pm \\
& 2 i \kappa t) \pm i), \text { or } \\
& \eta=a_{0}(1 \pm i \operatorname{coth}(\kappa(\mathrm{x} \pm 2 i \kappa t))) \pm \sqrt{\frac{2 a_{0}^{2}+\lambda^{2}}{2}}(\operatorname{coth}(\kappa(\mathrm{x} \pm \\
& 2 i \kappa t) \pm i),
\end{aligned}
$$

$$
\begin{aligned}
& \text { Case (2) } \\
& \kappa=\frac{\pi \lambda}{2}, b_{1}=0, a_{1}=-\frac{\lambda}{\sqrt{2}}, b_{0}=\frac{\pi \lambda}{\sqrt{2}}, a_{0}=0, \\
& \eta= \pm \sqrt{2} i \kappa(i \pm \tanh (\kappa(x \pm 2 i \kappa t))) \text {, or } \\
& \eta= \pm \sqrt{2} i \kappa(i \pm \operatorname{coth}(\kappa(x \pm 2 i \kappa t))),
\end{aligned}
$$

The solitary wave and behavior of the solutions $\varphi(\mathrm{x}, \mathrm{t})$ and $\psi(\mathrm{x}, \mathrm{t})$ are shown in Figure (3) for some fixed values of the parameters, $\left(a_{0}=1, \kappa=0.5\right)$
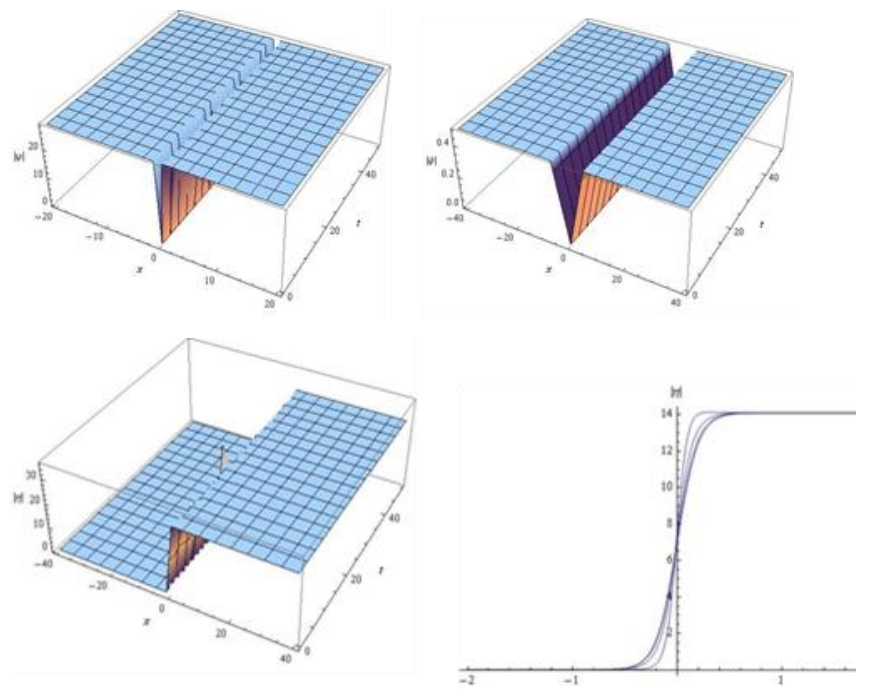

Fig 3: the component $|\varphi|$, the component $|\psi|$, and $|\eta|$ of the example(3)

\section{CONCLUSIONS}

The powerful tanh method was employed for analytic treatment of nonlinear coupled partial differential equations. The tanh method require transformation formulas. Traveling wave solutions, kinks solutions were derived.The performance of the tanh method show that these methods are reliable and effective. The applied methods will be used in further works to establish more entirely new solutions for other kinds of nonlinear equations.

\section{REFERENCES}

[1] Abdul-Majid Wazwaz. 2008 .Solitary wave solutions of the generalized shallow water wave (GSWW) equation by Hirota's method, tanh-coth method and Exp-Function method, Appl. Math. Comput. 202 275-286.

[2] Khater, A.H., Malfiet, W., Callebaut, D.K., Kamel, E.S. 2002, The tanh method, a simple transformation and exact analytical solutions for nonlinear reactiondiffusion equations. Chaos Solitons Fractals 14, 513-522

[3] Evans, D.J., Raslan, K.R. (2005), The tanh function method for solving some important nonlinear partial differential equation.Int. J. Comput. Math. 82(7), 897905

[4] Fan, E.2000, Extended tanh-function method and its applications to nonlinear equations. Phys. Lett. A 277 , 212-218

[5] Elwakil, S.A., El-Labany, S.K., Zahran, M.A., Sabry, R. 2002, Modified extended tanh-function method for solving nonlinear partial differential equations. Phys. Lett. A 299, 179-188

[6] Gao, Y.T., Tian, B. 2001, Generalized hyperbolicfunction method with computerized symbolic computation to construct the solitonic solutions to nonlinear equations of mathematical physics. Comput. Phys. Commun. 133, 158-164

[7] Tian, B., Gao, Y.T. 2002 Observable solitonic features of the generalized reaction diffusion model. Z Naturforsch. A $57,39-44$

[8] W.Malfliet, 1992,Solitary wave solutions of nonlinear wave equations, Am. J.Phys. (60)650-654.

[9] Abdul-Majid Wazwaz,2006,Exact and explicit travelling wave solutions for the nonlinear Drinfeld Sokolov system,Comm. Nonlinear Sci. Num.Siml.11(3):311-325.

[10] Abdul-Majid Wazwaz, (2006),Two reliable methods for solving variants of the $\mathrm{KdV}$ equation with compact and non-compact structures, Chaos Solitons Fract.28(2)454462 .

[11] Abdul-Majid Wazwaz,2006, The tanh method for compact and non-compact solutions for variants of the KdV-Burger equations, Phys. D: Nonlinear Phenomena 213 (2) 147-151.

[12] Hoseini,S. M. and Marchant, T. R., 2006 Solitary wave interaction and evolution for higher-order Hirota equation, Wave Motion. 44, pp. 92-106,

[13] WANG Q, CHEN Yong, LI Biao and ZHANG HongQing,2004,New exact travelling wave Solutions to Hirota equation and (1+1)-dimensional dispersive long wave equation Theor. Phys. (Beijing, China) (41 ). 821-828

[14] Ismail M S and Taha T R 2007 Math. Comput. Simul. 74 302 